\title{
PREVALENCIA, DIMENSIONES Y VÍAS DE ADQUISICIÓN EN EL MIEDO A VOLAR EN AVIÓN
}

\author{
MIQUEL TORTELLA-FELIU ${ }^{1}$ y MIQUEL A. FULLANA \\ ${ }^{1}$ Universidad de las Islas Baleares
}

(Recibido el 18 de febrero de 1999)

\begin{abstract}
El objetivo de este trabajo es el de presentar los resultados más destacados de un estudio epidemiológico sobre el miedo a volar en avión en una muestra de 523 personas de la población general. Sesenta y nueve sujetos, lo que supone un $13,19 \%$ de la muestra estudiada, puede considerarse que presentan dicha alteración. Un $14,42 \%$ de las personas estudiadas también manifiestan niveles de malestar significativos al viajar en avión aunque no puedan considerarse fóbicas. Respecto a las dimensiones del miedo, el motivo principal de temor es el de sufrir un accidente (22\%), seguido por el miedo a no tener control de la situación (13,6\%). Lo más habitual de todos modos, es que los sujetos con miedo indiquen diversos componentes como los responsables principales de su miedo a volar en avión $(45,8 \%)$. Estos resultados apuntan que el miedo a volar en avión no puede considerarse un miedo unitario. En cuanto a la instauración del trastorno más del cincuenta por ciento de los casos no se relacionan con ningún desencadenante claro de su inicio. Se discuten las implicaciones nosológicas y de indicación de tratamiento a partir de estos datos.
\end{abstract}

Palabras clave: Miedo a volar, fobias especificas, estudios epidemiológicos.

\section{Prevalence, main components and ways of acquisition of fear of flying}

In this study we examined the prevalence, components and ways of acquisition of fear of flying in a sample of 523 subjects from the general population. Subjects were given several questionnaires to determine intensity and history of the fear. Sixty-nine subjects $(13 \%)$ were fearful flyers and an additional $14 \%$ suffered important distress when flying but were not phobics. The main component of the fear of flying was the fear of having an accident (22\%) followed by the fear of not having control of the situation. Anyway, most of the subjects attributed their fear to more than one component. Regarding the ways of acquisition, in more than a $50 \%$ from the subjects the fear onset was not associated with any clear trigger. Our results suggest that fear of flying cannot be regarded as a unitary fear. Nosologic and treatment implications are discussed.

Key words: Fear of flying, specific phobias, epidemiological studies.

\section{INTRODUCCIÓN}

El miedo a volar en avión, en sus diversos grados, afecta aproximadamente a

Agradecimientos: Este trabajo se ha podido llevar a cabo, en parte, gracias al proyecto de investigación subvencionado por la Dirección General de Enseñanza Superior del Ministerio de Educación y Cultura del Gobierno Español "Evaluación de un programa informático para el tratamiento del miedo a volar" (PB96-0199). Queremos agradecer la colaboración de la Regidoria d'Educacio de l'Ajuntament de Palma en la realización de este estudio y a Rebeca Urrea y Antoni Rubí por su ayuda en la recogida de datos. una cuarta parte de la población general (Greco, 1989; Van Gerwen, Spinhoven, Diekstra y Van Dyck, 1997). En los casos más extremos, se trata de un problema que tiene importantes repercusiones negativas para quien lo padece. Por motivos poco conocidos, el miedo a volar en

Correspondencia: Miquel Tortella-Feliu, Dpto. de Psicología, Universidad de las Islas Baleares, Carretera de Valldemossa km. 7,5. Edificio Guillem Cifre de Colonia, 07071 Palma (Mallorca). Tfno.: 971 172 579. Fax 971173 190. dpsmtfo@ps.uib.es 
avión ha recibido una menor atención que otros miedos que no tienen, al menos en nuestro entorno, la importancia y repercusión socioeconómica que presenta este problema. Hasta hace tan solo unos años, la mayoría de trabajos sobre el miedo a volar eran estudios de tratamiento no controlados y, en general, el nivel global de la investigación estaba muy por debajo de lo esperable dado su alcance (Bornas y Tortella-Feliu, 1995). Recientemente han aparecido los primeros estudios epidemiológicos (Curtis, Magee, Eaton, Wittchen y Kessler, 1998; Fredrikson, Annas, Fisher y Wik, 1996) y descripciones clínicas (Van Gerwen et al., 1997; Wilhelm y Roth, 1997) de cierto rigor que nos han permitido avanzar notablemente en la conceptualización del trastorno. La investigación en este ámbito se ha centrado, básicamente, en tres aspectos: la determinación de las tasas de prevalencia; las dimensiones del miedo a volar -y por extensión, su ubicación diagnóstica-; y el análisis de sus vías de adquisición.

\section{Prevalencia}

En general, los datos epidemiológicos sobre el miedo a volar son poco precisos (Rubio, Cabezuelo y Castellano, 1996) y las cifras de prevalencia que se aportan se mueven en un rango excesivamente amplio. Así, por ejemplo, Van Gerwen et al. (1997), resumiendo los diferentes trabajos sobre este tema, señalan que la prevalencia del miedo a volar, en sus distintos grados, está entre el 10 y el $40 \%$ de la población general en países industrializados. Algunos años antes, Greco (1989) resumía los estudios publicados hasta la fecha afirmando que un $25 \%$ de la población presenta miedo cuando vuela y que el porcentaje de personas cuya evitación permite considerarlas como fóbicos es del $10 \%$.

Pocos han sido los trabajos epidemiológicos que hayan estudiado específicamente el miedo a volar. En la Tabla 1 se resumen los trabajos en que se ha hecho referencia a las tasas de prevalencia.

Tabla 1. Principales estudios epidemiológicos sobre el miedo a volar en población general

\begin{tabular}{|c|c|c|c|c|c|c|}
\hline Estudio & Ciudad/País & $N$ & Tipo de evaluación & Prevalencia puntu & & Prevalencia global \\
\hline Agras et al. (1969) & $\begin{array}{l}\text { Burlington } \\
\text { (EE.UU) }\end{array}$ & 325 & Entrevista estructurada & $\begin{array}{l}\text { Miedo moderado } \\
\text { Miedo intenso }\end{array}$ & $\begin{array}{l}20 \% \\
11 \%\end{array}$ & 一 \\
\hline Nordlund (1983) & Suecia & 2000 & & $\begin{array}{l}\text { Evitadores } \\
\text { Malestar moderado }\end{array}$ & $\begin{array}{l}10 \% \\
25 \%\end{array}$ & $\overline{-}$ \\
\hline Arnarson (1987) & Islandia & 1000 & & Miedo intenso & $17 \%$ & - \\
\hline Ekeberg et al. (1989) & Noruega & 790 & Cuestionario & $\begin{array}{l}\text { Evitadores } \\
\text { Siempre con miedo }\end{array}$ & $\begin{array}{r}5 \% \\
22 \%\end{array}$ & 一 \\
\hline Kendler et al. (1992) & $\begin{array}{l}\text { Virginia } \\
\text { (EE.UU.) }\end{array}$ & 2163 & Entrevista SCID & 一 & & $\begin{array}{l}\text { Fobia } \\
-\end{array}$ \\
\hline $\begin{array}{l}\text { Fredrikson et } \\
\text { al. (1996) }\end{array}$ & $\begin{array}{l}\text { Estocolmo } \\
\text { (Suecia) }\end{array}$ & 704 & $\begin{array}{l}\text { Cuestionario } \\
\text { (criterios DSM-IV) }\end{array}$ & Fobia & $2,6 \%$ & - \\
\hline $\begin{array}{l}\text { Tortella-Feliu et } \\
\text { al. (1996) }\end{array}$ & $\begin{array}{l}\text { Palma } \\
\text { (Mallorca) }\end{array}$ & 562 & Cuestionario & $\begin{array}{l}\text { Miedo a volar } \\
\text { Malestar moderado }\end{array}$ & $\begin{array}{r}9 \% \\
13 \%\end{array}$ & - \\
\hline Curtis et al. (1998) & EE.UU. & 8098 & Entrevista CIDI & 一 & & $\begin{array}{lr}\text { Miedo a volar } 13 \% \\
\text { Fobia } & 5 \%\end{array}$ \\
\hline
\end{tabular}


El punto de partida se sitúa en el estudio clásico de Agras, Sylvester y Oliveau (1969) realizado en población general sobre la prevalencia de los principales miedos y fobia. En este trabajo, un $10 \%$ de los sujetos interrogados informaba de intenso miedo a volar y un $20 \%$ adicional presentaba malestar moderado. El miedo a volar era el cuarto más frecuente de la muestra. De entre los trabajos realizados posteriormente queremos destacar especialmente el de Ekeberg, Seeberg y Ellersten (1989) en que se encontró que un $5 \%$ de sujetos de la población general evaluados evitaban volar y un $22 \%$ presentaba intenso malestar cuando lo hacían. En nuestro país, Tortella-Feliu, Bornas, Martínez Abascal y Urrea (1996) obtuvieron que el $9 \%$ de una muestra de 562 sujetos de población general tenían miedo a volar y que un $14 \%$ adicional presentaban cierto malestar cuando volaban.

El problema de la gran mayoría de los trabajos que aparecen resumidos en la Tabla 2 es que casi siempre se han basado en las respuestas a determinados cuestionarios y/o que no se han utilizado criterios diagnósticos precisos (Agras et al, 1969; Arnarson, 1987; Ekeberg et al., 1989; Kendler, Neale, Kessler, Heath y Eaves, 1992; Norlund, 1983; Tortella-Feliu, et al., 1996). Dos trabajos recientes superan esta limitación. Fredrikson et al. (1996), en un estudio en población general sueca y utilizando criterios DSM-IV, obtuvieron una prevalencia total puntual para la fobia específica (volar en avión) del $3 \%$. Por su parte, Curtis et al. (1998), en una muestra de más de 8.000 sujetos norteamericanos y utilizando entrevistas diagnósticas estructuradas, calcularon una prevalencia para toda la vida del 13 y del $5 \%$ para el miedo a volar y para la fobia a volar respectivamente.

\section{Dimensiones del miedo a volar}

Un aspecto de especial relevancia en el miedo a volar, y que también ha empe- zado a recibir una mayor atención últimamente, es el análisis de sus dimensiones. El estudio de Howard, Murphy y Clarke (1983) fue el primero que se ocupó de esta cuestión. Dentro del Cuestionario sobre las Actitudes hacia Volar (QAF, Questionnaire on Attitudes to Flying), uno de los instrumentos que se utilizó en la evaluación pretratamiento de los sujetos que acudieron a tratamiento para esta problemática, se incluyó una pregunta abierta respecto a qué era para ellos lo peor a la hora de tomar un avión. A partir del análisis de los contenidos de esas respuestas se definieron seis categorías o dimensiones principales del miedo a volar: miedo a tener un accidente, miedo a las alturas, miedo a la inestabilidad, miedo a experimentar sensaciones físicas desagradables-perder el control, miedo a permanecer en un lugar cerrado y miedo a estar en una situación de la que no se tiene control. Estas categorías son las que, con posterioridad, se han usado en la mayoría de trabajos sobre el tema.

Los trabajos posteriores han corroborado que el miedo a volar no es unitario (Greco, 1989; Howard et al., 1983; Tortella-Feliu et al., 1996; Van Gerwen et al., 1997; Wilhelm y Roth, 1997). Dada la heterogeneidad encontrada entre los sujetos con miedo a volar, algunos autores (Van Gerwen et al., 1997) han apuntado que este trastorno podría conceptualizarse, según los casos, bien como una fobia específica situacional bien como la expresión de otras fobias no situacionales e incluso como una alteración secundaria al trastorno de pánico con o sin agorafobia. La dificultad para encajar la fobia a volar dentro de las categorías tradicionales de comportamientos fóbicos ha hecho que en el manual más reciente y exhaustivo estos trastornos (Davey, 1997) se incluya en el apartado de fobias atípicas (McNally, 1997) 
Tabla 2. Dimensiones del miedo a volar en avión. Porcentaje de sujetos adscritos a cada categoría

\begin{tabular}{|c|c|c|c|c|c|c|}
\hline Dimensión & $\begin{array}{c}\text { Howard } \\
\text { et al. (1983) }\end{array}$ & $\begin{array}{c}\text { Walder } \\
\text { et al. (1987) }\end{array}$ & $\begin{array}{l}\text { Tortella-Feliu } \\
\text { et al. (1996) }\end{array}$ & $\begin{array}{c}\text { Öst } \\
(1996)\end{array}$ & $\begin{array}{l}\text { Van Gerwen } \\
\text { et al. (1997) }\end{array}$ & $\begin{array}{l}\text { Tortella-Feliu } \\
\text { et al. (1999) }\end{array}$ \\
\hline Tener un accidente & $52 \%$ & $34 \%$ & $72 \%$ & $43 \%$ & $25 \%$ & $25 \%$ \\
\hline Alturas & $23 \%$ & $13 \%$ & $4 \%$ & & $17 \%$ & $3 \%$ \\
\hline Lugar cerrado & $18 \%$ & $37 \%$ & $4 \%$ & $14 \%$ & $20 \%$ & $7 \%$ \\
\hline Inestabilidad & $5 \%$ & & $2 \%$ & & & $3 \%$ \\
\hline $\begin{array}{l}\text { No tener control } \\
\text { de la situación }\end{array}$ & $5 \%$ & & $10 \%$ & $18 \%$ & $20 \%$ & $7 \%$ \\
\hline Sensaciones físicas & & & & & & $3 \%$ \\
\hline Perder el control & $5 \%$ & & & & $7 \%$ & \\
\hline $\begin{array}{l}\text { Fuentes diversas / } \\
\text { otras fuentes }\end{array}$ & & $26 \%$ & & & $11 \%$ & $51 \%$ \\
\hline
\end{tabular}

Como puede apreciarse en la Tabla 2, en todos los estudios que han tratado esta cuestión, el temor a tener un accidente ha sido citada como la dimensión principal del miedo a volar (Howard et al.,1983; Öst, 1996; Tortella-Feliu et al., 1996; Van Gerwen et al., 1997) excepto en el de Walder, McCraken, Herver, James y Brewitt (1987), donde el miedo principal era a estar encerrado. Los resultados de estos trabajos muestran que las dimensiones (miedos) más importantes en el miedo a volar son, por este orden: a tener un accidente, a estar encerrado, a no tener control, a las alturas y a la inestabilidad. En el trabajo de Van Gerwen et al. (1997) las dimensiones del miedo fueron valoradas en función del sexo. La causa del miedo a volar más señalada por hombres era el miedo al accidente, mientras que en las mujeres era el miedo a no tener control.

\section{Vías de adquisición del miedo a volar}

Desde hace algún tiempo, y a partir del clásico estudio de Rachman (1977), se ha venido considerando que los miedos y fobias se pueden adquirir de forma directa (por condicionamiento clásico) o indi- recta (experiencias vicarias y transmisión de información). Numerosos estudios han intentado determinar el peso específico de estas tres vías de adquisición en el origen de las fobias y parece que existen diferencias en cuanto a la adquisición según el subtipo de fobia específica (véase Tortella-Feliu y Fullana, 1998). Existen algunos trabajos previos en que este aspecto ha sido estudiado en el miedo a volar. En un estudio de McNally y Louro (1992) en el que se comparaban sujetos que solicitaban tratamiento para el miedo a volar con trastorno de pánico o «solo" fobia específica, los pacientes con fobia específica informaban más frecuentemente que los pacientes con trastorno de pánico $(71 \%$ frente a $18 \%$ ) que la transmisión de información estaba implicada en el origen de su miedo; también más pacientes con fobia específica ( $41 \%$ frente a ninguno) citaban la exposición a acontecimientos traumáticos durante el vuelo como factor etiológico; y una mayor proporción de sujetos con fobia específica citaban la adquisición vicaria como predominante, aunque en este caso las diferencias no eran significativas.

Por su parte, Wilhelm y Roth (1997) dividieron una muestra de personas con 
miedo a volar en tres subgrupos: sujetos que presentaban trastorno de pánico con agorafobia; sujetos que tenían historia de trastorno de pánico con agorafobia; y sujetos que tenían "solo» fobia específica. Estos autores no encontraron diferencias entre los subgrupos en los porcentajes de sujetos que indicaban una u otra categoría (condicionamiento directo, vicario o transmisión de información) como predominante en el origen de su miedo. La atribución de la causa primaria de la fobia dependía de si los sujetos presentaban o no historia de trastorno de pánico. Los fóbicos sin historia de trastorno de pánico atribuían su miedo a acontecimientos traumáticos $(46 \%)$ y pocas veces a ataques de pánico $(11 \%)$.

En un estudio realizado en 84 personas con miedo a volar, Sosa, Capafóns y Viña (1993), informaron que el $53 \%$ de los sujetos atribuían la instauración del problema a una experiencia directa, el $11 \%$ a una experiencia indirecta y el $34.5 \%$ no podía especificarlo.

El presente estudio tiene tres objetivos. En primer lugar, investigar la prevalencia del miedo a volar en Mallorca. En segundo lugar, determinar la importancia relativa de las diferentes dimensiones del miedo a volar. Siguiendo investigaciones previas sobre este tema (Howard et al., 1983; Tortella-Feliu et al, 1996; Van Gerwen et al., 1997; Wilhelm y Roth, 1997) se hipotetizó que la dimensión principal del miedo a volar sería el miedo a tener un accidente. El último objetivo era estudiar qué vías de adquisición son predominantes en el miedo a volar. Se esperaba que las tres vías de adquisición "clásicas» (condicionamiento directo, condicionamiento vicario e información/instrucción) estarían implicadas en el miedo a volar. Debido a que los trabajos anteriores relativos a esta cuestión ofrecen datos poco concluyentes, no existían expectativas previas sobre la predominancia de una u otra vía de adquisi- ción que nos permitieran establecer una hipótesis formal al respecto. En cualquier casos, en los estudios llevados a cabo en poblaciones clínicas sobre las vías de adquisición de las fobias situacionales (Himle, Crystal, Curtis y Fluent, 1991; Öst y Hughdal, 1981), aparte del ya citado de McNally y Louro (1992) en pacientes con fobia a volar, existe una clara superioridad de la instauración a través del condicionamiento clásico.

\section{MÉTODO}

\section{Sujetos}

La muestra la componen 523 sujetos de la población general de Palma (Mallorca) que asistían a cursos de formación para adultos organizados por el Ayuntamiento de la ciudad. En el momento de realizar el estudio, estaban matriculadas en estos cursos un total de 832 personas con diversos niveles educativos y socioeconómicos y, por tanto, la muestra parece reflejar adecuadamente las características de la población de origen. La edad media de la muestra era 31.6 años (rango 18-83; $\mathrm{DT}=9,29)$. El $74 \%$ eran mujeres. Únicamente 6 sujetos (un 1,15\%) de la muestra no habían volado nunca y la media de vuelos anuales autoinformados fue de $4,55(\mathrm{DT}=8,93)$

\section{Medidas}

Cuestionario de Miedo a Volar-II (Qüestionari de por de volar-II, QPV-II, Bornas y Tortella-Feliu, García de la Banda, Fullana y Llabrés, en prensa). El QPV-II, elaborado originalmente en catalán, es un listado de 30 situaciones relacionadas con volar en avión y que pueden generar malestar o ansiedad, divididas en tres subescalas: situaciones durante el vuelo, previas al vuelo y vicarias. Los items (cada 
uno representa una situación) se valoran en escalas Likert de 1 (malestar mínimo) a 10 (malestar máximo) y, por tanto, el rango de puntuación está entre 30 y 300 . El QPV-II es una versión ligeramente modificada del Cuestionario de Miedo a Volar (Qüestionari de por de volar, QPV, Bornas y Tortella-Feliu, 1995). Los resultados de la validación factorial del QPV (Bornas et al., en prensa) nos llevaron a una depuración del instrumento que dio lugar a esta nueva versión. Se excluyeron los items 3 , $24,29,33$ y 34 de la primera versión. Los items 26 y 22 se unificaron en uno solo, se reformulo el enunciado de los items 7 y 19 y se añadieron dos nuevos items. El instrumento aparece reproducido en Bornas et al. (en prensa). El QPV-II presenta una elevada consistencia interna $a=.96 \mathrm{y}$ también posee un alto grado de validez de criterio, atendiendo a su capacidad para discriminar entre sujetos fóbicos, no fóbicos y muestras subclínicas (Bornas et al., en prensa).

Historia Personal de Vuelo (TortellaFeliu y Bornas, 1995). Es un cuestionario estructurado que recoge información sobre los factores de instauración y mantenimiento del miedo a volar en avión y sus dimensiones, manifestaciones psicofisiológicas durante el vuelo, presencia de conductas de evitación y su intensidad (valorada en una escala Likert de 1 a 10, donde 1 representa una evitación mínima y 10 una evitación máxima), prevalencia del miedo en personas del entorno inmediato del sujeto y presencia de otros miedos fóbicos. Respecto a las dimensiones del miedo, se solicita a la persona que indique cuál o cuales de los siguientes factores se relaciona/n más con su miedo a volar: miedo a las sensaciones físicas, a tener un accidente, a las alturas, a encontrarse en un lugar cerrado, a la inestabilidad y relacionado con no tener control de la situación. Tales factores corresponden a las dimensiones más frecuentemente obtenidas en ante- riores estudios sobre esta cuestión (Howard et al., 1983; Tortella-Feliu et al., 1996; Van Gerwen et al., 1997; Wilhem y Roth, 1997). También se pregunta al participante que valore, en una escala Likert de 0 (mínimo) a 10 (máximo), el grado de malestar que le produce cada uno de estos factores. Otro aspecto evaluado en el cuestionario era el momento de aparición del miedo a volar, que constaba de tres posibles categorías: (1) el miedo ha existido desde siempre -antes de tomar el primer vuelo en avión-; (2) apareció después de haber realizado el primer vuelo; y (3) surgió después de llevar un tiempo volando sin problemas. Finalmente, en cuanto a las vías de adquisición, se preguntaba al participante si recordaba alguna situación que hubiera desencadenado su miedo a volar y se le ofrecían seis posibles opciones, referidas respectivamente a la adquisición por condicionamiento directo (vuelo complicado, accidente leve, etc.); por condicionamiento vicario (ver a alguien con mucho miedo en el avión o antes de cogerlo); por instrucción (alguien cercano comenta haber tenido un mal vuelo); y por información (leer noticias de accidentes de aviación). Las otras dos opciones eran «haber tenido miedo desde siempre» y no recordar ningún desencadenante (el miedo a volar comenzó después de volar durante algún tiempo sin miedo). Este cuestionario, que no ha sido publicado, se encuentra disponible en http://www.uib.es/depart/dpsweb/petra.html.

Escala de malestar global. Valoración en una escala Likert de 1 (mínimo) a 10 (máximo) del malestar global experimentado ante el hecho de tener que tomar un avión.

\section{Procedimiento}

La recogida de datos se realizó entre los días 24 de noviembre y 12 de diciem- 
bre de 1997. Uno de los miembros del equipo de investigación acudía al aula donde se realizaba el curso, explicaba los objetivos del estudio al grupo y atendía individualmente las posibles dudas surgidas al cumplimentar los cuestionarios. Cada uno de los participantes recibía un cuadernillo que contenía los instrumentos arriba señalados.

\section{RESULTADOS}

\section{Prevalencia del miedo a volar en avión}

Un total de 69 sujetos [12 hombres $(17,4 \%)$ y 57 mujeres $(82,6 \%)]$ manifestaron tener miedo a volar (contestaron "Sí» a la pregunta " ¿considera Vd. que tiene miedo a volar?»). Esto representa un $13,19 \%$ del total de la muestra. La media de la edad de los sujetos con miedo a volar $(M=35,7 ; D T=9,70)$ era superior a la de los sujetos sin miedo $(\mathrm{M}=32,14, \mathrm{DT}=9,14)$ y esta diferencia era estadísticamente significativa $(t=2,99$, $\mathrm{p}<0,003)$. Todos ellos dieron una puntuación igual o superior 4 en la escala de malestar global. No consideramos oportuno la inclusión de un criterio más restrictivo para la inclusión de los sujetos dentro del grupo de miedo a volar (como hubiera podido ser el del grado de evitación) porque dadas las condiciones geográficas y sociales del lugar donde se ha llevado a cabo el estudio, resulta muy difícil evitar el hecho de tomar un avión. Muchas de las personas con miedo, que probablemente en otros lugares evitarían de una forma mucho más amplia esta situación, aquí se exponen a ella aunque sea a costa de un elevado malestar.

Se utilizó una puntuación igual o superior a 90 en QPV-II como punto de corte para definir que un sujeto presentaba malestar moderado al volar. Considerando este criterio, un $14,42 \%$ de los sujetos que manifestaron no tener miedo a volar presentaban malestar moderado al volar en avión. Respecto a las variables relacionadas con la intensidad del miedo que se han contemplado en este trabajo, aparecen diferencias estadísticamente significativas en todas ellas entre el grupo de sujetos con miedo a volar y los que dicen no padecerlo (véase Tabla 3).

\section{Dimensiones del miedo}

A partir de las respuestas a la Historia Personal de Vuelo, los sujetos con miedo a volar fueron clasificados según la dimensión predominante en su miedo. En los casos en que el sujeto señalaba más de una dimensión, se consideraba como principal la de mayor puntuación. Cuando la puntuación más alta se daba en más de una dimensión al mismo tiempo, el sujeto era adscrito a la categoría "varias dimensiones».

Se calcularon las frecuencias y porcentajes de sujetos que se incluían en cada una de las seis dimensiones antes citadas, así como sus medias en intensidad. En 10 de los casos no se obtuvieron datos váli-

Tabla 3. Comparación de medias en las variables relacionadas con la intensidad del miedo entre los sujetos con y sin miedo a volar autoinformado

\begin{tabular}{lrrrrr}
\hline & \multicolumn{2}{l}{ Ss con miedo a volar } & \multicolumn{2}{c}{ Ss sin miedo a volar } & \multirow{2}{*}{ DT } \\
& Media & DT & Media & DT & \\
\hline QPV-II & 159,41 & 45,51 & 65,24 & 30,80 & $21,89^{\star}$ \\
Malestar global & 7,30 & 1,85 & 1,36 & 1,36 & $27,64^{*}$ \\
Evitación & 3,65 & 3,60 & 0,19 & 0,87 & $17,30^{*}$ \\
\hline
\end{tabular}

Nota: ${ }^{*} p=0,000$ 
dos y, por tanto el análisis de resultados se realizó sobre un total de 59 sujetos. Existen diferencias estadísticamente significativas entre las dimensiones del miedo autoinformadas $\left(\chi^{2}(6)=60,24\right.$, $\mathrm{p}=0,000$ ). Como puede verse en la Tabla 4 , el temor que aparece citado por un mayor número de sujetos como motivo principal del miedo a volar en avión es el de sufrir un accidente ( $22 \%$ ), seguido por el miedo a no tener control de la situación $(13,6 \%)$. Estos dos temores son también los citados con más frecuencia por todos los sujetos (sea o no el miedo más importante) y son además los que obtienen una valoración media en intensidad más elevada $(6,52$ y 5,85 respectivamente).

La mayoría de personas interrogadas señalaban varios componentes en su miedo a volar y los valoraban con la misma intensidad. Entre los sujetos que citaban varios componentes, los más citados fueron, de nuevo, el miedo a los accidentes, señalado por 22 de los 27 sujetos ( $81 \%$ ) $y$ el hecho de encontrarse en una situación en la que no se tiene control, señalado por 21 (78\%). En 18 casos (67\%) el miedo a los accidentes y a no tener control de la situación fueron citados conjuntamente. El resto de factores fueron citados en muy menor medida $y$, como norma general, siempre en sujetos que citaban más de dos factores como dimensiones del miedo. Así, 10 personas (37 $\%$ ) citaban el miedo a la inestabilidad como uno de los factores principales e igual número el miedo a las alturas. Seis
(22\%) citaron el miedo a permanecer en un lugar cerrado del que puede ser difícil escapar y únicamente cinco sujetos (19\%) mencionaron el temor a experimentar sensaciones físicas desagradables como elemento prioritario del miedo.

Relación de las dimensiones con la intensidad del miedo a volar

Con el objeto de analizar la relación entre las dimensiones del miedo a volar y las variables referidas a su intensidad, se calcularon las puntuaciones medias en el QPV-II, la escala de valoración del malestar global y la escala que valoraba la intensidad de la evitación, según la dimensión citada como responsable principal del miedo. Como puede apreciarse en la Tabla 5, los sujetos que citan diversas fuentes como dimensión principal del miedo son los que presentan puntuaciones más elevadas en los tres tipos de medida utilizadas en la comparación. Los sujetos con miedo a las alturas son los que, después de quienes informan de diversas fuentes, presentan puntuaciones más elevadas en el QPV-II y en malestar global. Con respecto a las conductas de evitación, se sitúan en tercer lugar por detrás de los que manifiestan temor a no tener control de la situación como dimensión principal de su conducta problema.

Dado el escaso número de sujetos por categoría el análisis se realizó sobre las

Tabla 4. Dimensiones del miedo a volar el avión ( $n=59$ )

\begin{tabular}{lccc}
\hline \multicolumn{1}{c}{ Dimensión } & $\begin{array}{c}\text { Frecuencia de } \\
\text { aparición }\end{array}$ & $\begin{array}{c}\text { Lo señalan como } \\
\text { miedo principal }\end{array}$ & $\begin{array}{c}\text { Intensidad } \\
M(D T)\end{array}$ \\
\hline Miedo a las sensaciones físicas & 30 & $1(1,7 \%)$ & $3,62(3,18)$ \\
Miedo a los accidentes & 52 & $13(22 \%)$ & $6,52(3,18)$ \\
Miedo a las alturas & 35 & $5(8,5 \%)$ & $4,26(3,74)$ \\
Miedo a las alturas & 36 & $4(6,8 \%)$ & $4,55(3,62)$ \\
Miedo a la inestabilidad & 38 & $1(1,7 \%)$ & $4,52(3,47)$ \\
Miedo a no tener control & 43 & $8(13,6 \%)$ & $5,85(3,72)$ \\
Diversas fuentes & & $27(45,8 \%)$ & \\
\hline
\end{tabular}


Tabla 5. Dimensión principal del miedo a volar en avión e intensidad de las manifestaciones fóbicas

\begin{tabular}{lcccc}
\hline \multicolumn{1}{c}{ Dimensión } & Miedo principal & QPV-II & Malestar global & Evitación \\
\hline Miedo a las sensaciones físicas & $1(1,7 \%)$ & 157,00 & $\mathbf{8 , 0 0}$ & 2,00 \\
Miedo a los accidentes & $13(22 \%)$ & 140,66 & 7,25 & 2,33 \\
Miedo a las alturas & $5(8,5 \%)$ & 177,00 & 7,50 & 3,20 \\
Miedo a las alturas & $4(6,8 \%)$ & 128,50 & 6,00 & 1,50 \\
Miedo a la inestabilidad & $1(1,7 \%)$ & 109,00 & 6,00 & 0,00 \\
Miedo a no tener control & $8(13,6 \%)$ & 144,25 & 6,88 & 4,63 \\
Diversas fuentes & $27(45,8 \%)$ & 187,34 & 8,17 & 4,78 \\
\hline
\end{tabular}

Nota: No información: 10 sujetos

categorías siguientes: miedo a los accidentes, alturas, lugares cerrados y no control. Se formó una nueva categoría ("diversas fuentes») que incluía las restantes. La prueba de Kruskal-Wallis determinó que las diferencias entre grupos eran significativas en las puntuaciones del QPV-II $\left(\chi^{2}=15,19, p=0,004\right)$, pero no en las escalas de malestar global $\left(\chi^{2}=7,02\right.$, $\mathrm{p}=0,135)$ y evitación $\left(\chi^{2}=6,46, p=0,167\right)$.

\section{Vías de adquisición}

Los datos referidos al momento de inicio y a las vías de adquisición del miedo a volar también se obtuvieron a partir de las respuestas a la Historia Personal de Vuelo (véase apartado «Medidas»).

En cuanto al momento de inicio, de los 58 sujetos de los que se dispone de datos, $17(29,3 \%)$ manifestaron que su miedo existía desde siempre, incluso antes de tomar el primer vuelo, 13 (22,4\% del total) indicaron que el miedo apareció justo des- pués del primer vuelo llevado a cabo y 28 $(48,3 \%)$ manifestaron que el miedo aparecí después de un tiempo en que volaron sin experimentar ningún tipo de malestar destacable. Entre los sujetos que podían aislar el momento en que empezaron a tener miedo, la edad media de inicio era de 20,17 años (rango 4-36; DT=7,20).

Respecto a las vías de adquisición, en primer lugar, se han calculado las frecuencias y porcentajes de sujetos que citaron alguna de las tres vías de adquisición apuntadas por Rachman (1977) y en las que, con posterioridad, se han centrado la mayoría de estudios sobre la etiología de los comportamientos fóbicos. En este primer análisis las categorías se han considerado como no excluyentes, es decir, que una persona puede haber citado más de una vía de adquisición y por tanto se recoge la frecuencia de aparición de esa vía en las valoraciones retrospectivas de instauración.

Como puede observase en la Tabla 6 , $16(26,7 \%)$ sujetos citan una experiencia

Tabla 6. Vías de adquisición del miedo a volar e intensidad de las manifestaciones fóbicas $(n=60)$

\begin{tabular}{|c|c|c|c|c|c|}
\hline Vía de adquisición & $\begin{array}{c}\text { Frecuencia } \\
\text { de aparición } \\
\text { Ss (\%) }\end{array}$ & $\begin{array}{c}\text { Via de } \\
\text { adquisición } \\
\text { principal } \\
\text { Ss (\%) }\end{array}$ & $\begin{array}{l}Q P V-I I \\
M(D T)\end{array}$ & $\begin{array}{l}\text { Malestar } \\
\text { global } \\
M(D T)\end{array}$ & $\begin{array}{c}\text { Grado de } \\
\text { evitación } \\
M(D T)\end{array}$ \\
\hline $\begin{array}{l}\text { Condicionamiento directo } \\
\text { Condicionamiento vicario }\end{array}$ & $\begin{array}{c}16(26,7 \%) \\
4(6,7 \%)\end{array}$ & $\begin{array}{l}8(13,3 \%) \\
0(0 \%)\end{array}$ & $160,00(39,92)$ & $8,50(, 93)$ & $4,38(3,74)$ \\
\hline Información/instrucción & $19(31,7 \%)$ & $10(16,7 \%)$ & $153,40(54,58)$ & $6,40^{-}(2,41)$ & $2,60(3,20)$ \\
\hline No recuerda & - & $33(55 \%)$ & $154,81(46,64)$ & $7,10(1,86)$ & $3,18(3,62)$ \\
\hline Varias vías & - & $9(15 \%)$ & $182,55(43,70)$ & $8,00(1,93)$ & $5,44(3,94)$ \\
\hline
\end{tabular}


aversiva directa como desencadenante del miedo a volar, $19(31,7 \%)$ lo asocian a la transmisión de información, bien por parte de otras personas cercanas bien a través de los medios de comunicación, y solamente en 4 casos $(6,7 \%)$ se cita una experiencia vicaria como hecho relacionado con el inicio del miedo $\left(\chi^{2}(2)=\right.$ $9,69, p=, 008$ ). Destaca el hecho de que 33 personas, un $55 \%$ de los sujetos de los que se dispone de datos, no recuerdan o no son capaces de aislar ninguna de las circunstancias recogidas en estas tres categorías como inicio de su miedo.

En segundo lugar, se ha prestado atención al número de sujetos con miedo a volar que han informado de una única o de diversas vías de adquisición. Solamente18 personas, un $30 \%$, de las 60 personas de las que se dispone de datos, aíslan una única vía de adquisición: 8 de ellas $(13,3 \%)$ un acontecimiento aversivo directo y $10(17,2 \%)$ la instrucción-transmisión de información. Ninguna persona hace referencia a una experiencia vicaria como único factor determinante de la instauración de su miedo. Los cuatro casos que habían citado esa vía lo hacen en combinación con otros factores, bien la transmisión de información bien una experiencia aversiva directa. En total son 9 (un $15 \%$ ) las personas que citan diversas vías a la vez como factores de adquisición del miedo a volar que experimen$\tan$ en la actualidad. No existen diferencias estadísticamente significativas respecto a las vías de adquisición autoinformadas $\left(\chi^{2}(2)=0,222 p=0,895\right.$ n.s.), si bien en este análisis no se tiene en cuenta el valor 0 de la casilla de condicionamiento vicario. Como ya se ha dicho anteriormente, más de la mitad de los sujetos no informan de ningún factor desencadenante del miedo a volar.

En cuanto a la relación entre las vías de adquisición y la intensidad del miedo, los diferentes análisis de la variancia realizados revelan que no existen diferen- cias estadísticamente significativas en función del modo de adquisición, ni en las puntuaciones en el QPV-II $(F=, 89$, $\mathrm{p}=, 452 \mathrm{n} . \mathrm{s}$.), ni en el grado de evitación $(F=1,30, p=, 285)$, ni tampoco en el nivel de malestar global $(F=2,31, p=, 087)$.

\section{DISCUSIÓN}

En el presente estudio, un $13 \%$ de los sujetos de una muestra de la población general afirmaron tener miedo a volar. A pesar de la gran limitación que supone haber considerado sujetos con miedo a aquellos que simplemente respondían que sí a una pregunta en que se les interrogaba al respecto y a que el grado de malestar autoinformado fuera igual o superior a 4, se trata de un porcentaje de la muestra considerable. Estudios anteriores (Agras et al. 1969; Dean y Whitaker, 1980; Öst, 1996) habían obtenido valores similares, aunque recientes trabajos sobre la prevalencia de las fobias específicas en población general arrojaron cifras sensiblemente inferiores (Curtis et al., 1998; Fredrikson et al., 1996). Lo más probable es que estas diferencias sean debidas, precisamente, a la utilización de criterios diferentes en la clasificación de los sujetos. Los resultados de éste y anteriores estudios indican que, aunque el miedo a volar es frecuente entre la población general, el porcentaje de sujetos en quienes se cumplen los criterios que permiten dar un diagnóstico formal de fobia específica (volar en avión) es mucho menor. Nuestra experiencia clínica con este grupo de pacientes nos induce a pensar que la fobia a volar es una de las fobias específicas más difíciles de diagnosticar utilizando los criterios nosológicos actuales (y, por tanto, una de las fobias con menor fiabilidad diagnóstica) dada la dificultad que supone establecer los parámetros de interferencia y evitación que determinan su diagnostico. 
También nos parece destacable reseñar que la tasa de prevalencia obtenida en nuestro estudio $(13,1 \%)$ es superior a la que hallamos, utilizando el mismo criterio, en un trabajo anterior $(9,1 \%)$ realizado en una muestra con una edad media casi diez años menor a la del presente trabajo (Tortella-Feliu et al., 1996). Consideramos que este hecho está motivado, precisamente, por la edad de los sujetos incluidos en las dos muestras, teniendo en cuenta que la edad media de inicio del miedo a volar en avión, como sucede para el conjunto de fobias específicas situacionales, sigue un patrón bimodal (infancia y principio de la edad adulta), con edades medias de inicio entre los 20 y 25 años, más tardías que para el resto de fobias específicas. La media de la edad de inicio del miedo a volar obtenida $(20,17$ años) es similar a la de un anterior trabajo (Tortella-Feliu et al, 1996). Aparte del porcentaje de sujetos que afirmaron tener miedo a volar, un porcentaje significativo adicional (más del $14 \%$ ) manifestó experimentar malestar en situaciones relacionadas con volar en avión. Esta circunstancia ya se había constatado en trabajos anteriores como el de Ekeberg et al. (1989) aunque las cifras obtenidas en este trabajo fueron algo superiores (un $22 \%$ ). En cualquier caso, es importante reconocer las limitaciones del presente estudio, que solo permite concluir que el $13 \%$ de la población estudiada considera tener miedo a volar en avión, sin tener en cuenta otros parámetros como la evitación o interferencia producidas por el miedo.

El segundo objetivo era determinar la importancia relativa de las diferentes dimensiones del miedo a volar. Nuestros datos muestran que el miedo a volar no es unitario, confirmando así los resultados de trabajos anteriores (Howard et al., 1983; Tortella-Feliu et al., 1996; Van Gerwen et al, 1997; Walder et al., 1987; Wilhelm y Roth, 1997). La hipótesis de que el miedo a tener una accidente sería la dimensión principal del miedo a volar fue confirmada, corroborando los resultados de todos los trabajos anteriores en que se había examinado esta cuestión (Howard et al., 1983; Tortella-Feliu et al., 1996; Van Gerwen et al, 1997 y Wilhelm y Roth, 1997) con la excepción del estudio de Walder et al. (1987) donde el miedo principal era a estar encerrado. El segundo miedo más importante en nuestro estudio era a no tener control. Este orden (miedo a tener un accidente en primer lugar seguido del miedo a no tener control) coincide con los resultados que habíamos obtenido previamente (Tortella-Feliu et al., 1996), aunque existen notables diferencias en el porcentaje de sujetos adscritos a cada categoría. Se encontró tambión una asociación entre las dimensiones del miedo y la intensidad de las manifestaciones fóbicas: existían diferencias estadísticamente significativas en el nivel de malestar autoinformado en el QPV-II en función del miedo principal, aunque no es así en las variables malestar general y grado de evitación. Las personas que informan de diversos miedos son las que exhiben puntuaciones más elevadas. De todos modos, los análisis realizados son muy limitados debido al escaso número de sujetos en cada categoría y por el hecho de haber forzado la agrupación de las dimensiones del miedo con menos de 5 sujetos por categoría.

La cuestión de la predominancia de una u otra dimensión en el miedo a volar y de cuál es la mejor manera de evaluar este aspecto merece ser investigada en posteriores estudios ya que posiblemente afecte a su ubicación nosológica y tiene importantes repercusiones terapéuticas. Posiblemente la conceptualización como fobia específica tipo situacional de aquellos casos en que predomine el miedo a tener un accidente sea la más adecuada. Sin embargo, en personas en que predomine el miedo a estar encerrado (o, por ejemplo, el miedo a las alturas) el miedo a volar es 
mejor entendido como la expresión de otros subtipos de fobias. Por último, algunos casos en que predomina el miedo a no tener control o a las sensaciones físicas desagradables podrían estar más próximos al pánico/agorafobia que a las fobias específicas. De hecho, algunos autores consideran que el miedo a volar en avión es una forma moderada de agorafobia (Himle et al., 1991). Este es, pues, un aspecto sobre el cual debe insistir la investigación sobre el miedo a volar en avión.

Un tercer objetivo del estudio era determinar qué vías de adquisición predominan en el miedo a volar. Algunos de los trabajos más destacados en el análisis de la epidemiología y origen de los comportamientos fóbicos (Fredrikson et al., 1996; Merckelbach, De Jong, Muris y van den Hout, 1996) han apuntado que en las fobias de tipo situacional es donde más claramente se aprecia el papel de las experiencias aversivas directas en la instauración del trastorno. Con respecto al miedo a volar en avión los datos son, hasta la fecha, escasos y poco concluyentes. En nuestro estudio destaca especialmente, como ya había sucedido en algún trabajo anterior (Sosa, Capafóns y Viña, 1993), el importante número de sujetos que no recuerda ningún incidente o incidentes como responsables del origen de su miedo y que un porcentaje destacable (por encima del $25 \%$ de la muestra) consideraba que padecía este temor desde siempre, incluso antes de haber tomado un avión. La categoría etiológica «siempre igual" o "desde siempre» fue incluida en el cuestionario de Menzies y Clarke (1993) para el análisis retrospectivo de la instauración de las fobias, a partir de las críticas a la estructura y contenido del cuestionario utilizado por Öst y Hughdal (1981), en el sentido que presentaba un claro sesgo hacia una categorización asociativa de las experiencias relacionas con los miedos fóbicos y que difícilmente podía contribuir a la contrastación de las teorías no asociacionistas (Menzies y Clarke, 1994).

De igual modo, resulta llamativo que ningún sujeto haya atribuido de forma exclusiva a una experiencia vicaria la responsabilidad principal en la adquisición del problema. Las personas que citan dicha circunstancia lo hacen siempre en combinación con alguna de las otras dos categorías contempladas.

Por otra parte, la distribución de sujetos en las otras dos vías de adquisición, junto con los que informan por igual de la incidencia de diversas fuentes, no presenta diferencias significativas.

La intensidad del miedo no aparece relacionada con el modo de instauración del problema, aunque en el caso del nivel de malestar global los resultados obtenidos se aproximan a la significación estadística, siendo los sujetos que informan de una experiencia aversiva directa para el inicio del miedo los que obtienen puntuaciones algo más elevadas que los restantes grupos. Por tanto no se cumple lo apuntado en el trabajo pionero de Rachman (1977), y que nunca ha podido ser claramente verificado, de que la intensidad del miedo se encuentra positivamente relacionada con la instauración a través de experiencias aversivas directas.

\section{REFERENCIAS BIBLIOGRÁFICAS}

Agras, S., Sylvester, D., y Oliveau, D. (1969). The epidemiology of common fears and phobias. Comprehensive Psy. chiatry, 10, 151-156.

Antony, M.M., Brown, T.A. \& Barlow, D.H. (1997).Heterogeneity among specific phobia types in DSM-IV. Behaviour, Research and Therapy, 35, 1089-1100.

Arnarson E.O. (1987). The prevalence of flight phobia among Icelanders. Comunicación presentada en el First Nordic Meeting, Aviation and Space Medicine. Oslo (Noruega). 
Bornas, X., y Tortella-Feliu, M. (1995) Descripción y análisis psicométrico de un instrumento de autoinforme para la evaluación del miedo a volar. Psicología Conductual, 3, 67-86.

Bornas, X., Tortella-Feliu, M., García de la Banda, G., Fullana, M.A., y Llabrés, J. (en prensa). Validación factorial del Cuestionario de Miedo a Volar. Análisis y Modificación de Conducta.

Curtis, G.C., Magee, W.J., Eaton, W.W., Wittchen, H.U. \& Kessler, R.C. (1998).Specific fears an phobias: Epidemiology and classification. British Journal of Psychiatry, 173, 212-217.

Davey, G.C.L. (Ed.) (1997). Phobias. A Handbook of theory, research and treatment. Chichester: Wiley.

Dean, R.D., y Withaker, K.M. (1980). Fear of flying: impact on the U.S. air travel industry. Boeing Company Document, BCS-00009-RO/OM.

Ekeberg, O., Seeberg, I., y Ellersten, B.B. (1989). The prevalence of flight anxiety in Norwy. Nordisk Psykiatrisk Tidsskrift, 43, 443-448.

Fredrikson, M. , Annas, P., Fischer, H., y Wik, G. (1996). Gender and age differences in the prevalence of specific fears and phobias. Behaviour Research and Therapy, 34, 33-39.

Greco, T.S. (1989). A cognitive.behavioral approach to fear of flying: a practitioner's guide. Phobia Practice and Research Journal, 2, 3-15.

Himle, J.A., Crystal, D., Curtis, G., y Fluent, T.E. (1991). Mode of Onset of Simple Phobia Subtypes: Further evidence of Heterogeneity. Psychiatry Research, 36, 37-43.

Howard, W.A., Murphy, S.M., y Clarke, J.C. (1983). The nature and treatment of fear of flying: a controlled investigation. Behavior Therapy, 14, 567567.

Kendler K.S., Neale M.C., Kessler R.C., Heath A.C., y Eaves L.J. (1992)The genetic epidemiology of phobias in women. Archives of General Psychiatry, 49, 273-281.

McNally, R.J. (1997). Atypical phobias. En G.C.L. Davey (Dir.). Phobias. A handbook of theory, research and treatment. (pp.183-199).Chichester: Wiley.

McNally, R.J., y Louro, C.E. (1992). Fear of flying in agoraphobia and simple phobia: distinguishing features. Journal of Anxiety Disorders, 6, 319-324.

Menzies, R.G., y Clarke, C. (1994). Retrospective studies of the origins of phobias: a review. Anxiety, Stress and Coping, 7, 305-318.

Merckelbach, H., De Jong, P, Muris, P., y van den Hout, M.A. (1996). The etiology of specific phobias: a review. Clinical Psychology Review, 16, 337-361.

Nordlund, C.L. (1983). Flygrädsla i Sverige. En enkätundersökning av svenskars rädsla för trafikflyg som transportmedel. Scandinavian Journal of Behavior Therapy, 12, 150-168.

Öst, L-G. (1996, octubre). Chair speakFast track treatment: exploring travel fears and changes during therapy. Comunicación presentada al $26^{\circ}$ Congreso de la European Association for Behaviour and Cognitive Therapy (EABCT). Budapest (Hungría)

Öst, L-G., y Hughdal, K. (1981). Acquisition of phobias and anxiety response patterns in clinical patients. Behaviour Research and Therapy, 19, 439-447.

Rachman, S. (1977). The conditioning theory of fear-acquisition: a critical examination. Behaviour Research and Therapy, 15, 375-387.

Rubio, V., Cabezuelo, F.J y Castellano, M.A. (1996). El miedo a volar en avión. Madrid: Biblioteca Nueva- Psicología Universidad.

Sosa, C.D., Capafóns, J.I., y Viña, C. (1993). Estudio descriptivo de la fobia a volar a través de la IDG-FV. Comunicación presentada al XXIV Congreso Iberoamericano de Psicología, Santiago de Chile. 
Sosa, C.D., Capafóns, J.I., Viña, C.M., y Herrero, M. (1995). La evaluación del miedo a viajar en avión: estudio psicométrico de dos instrumentos de tipo autoinforme. Psicología Conductual, 3 , 133-157.

Tortella-Feliu, M., y Bornas, X. (1995). Historia Personal de Vuelo. Manuscrito no publicado.

Tortella-Feliu, M., y Fullana, M.A. (1998). Una revisión de los estudios retrospectivos sobre el origen de las fobias específicas". Psicología Conductual; 6, 555-580.

Tortella-Feliu, M., Fullana, M.A., y Bornas, X. (1999). Características clínicas de la fobia a volar en avión. Manuscrito no publicado.

Tortella-Feliu, M., Bornas, X., MartínezAbascal, M.A., y Urrea, R. (1996, octu- bre). Ways of acquisition of fear of flying. Comunicación presentada al $26^{\circ}$ Congreso de la European Association for Behaviour and Cognitive Therapy (EABCT). Budapest (Hungría)

Van Gerwen, L.J., Spinhoven, P., Diekstra, R.F., y Van Dyck, R. (1997). People who seek help for fear of flying: typology of flying phobics. Behavior Therapy, 28, 237-251.

Walder, C.P., McCraken, J.S., Herbert, M., James, P.T., y Brewitt, N. (1987). Psychological intervention in civilian flying phobia: evaluation and a threeyear follow-up. British Journal of Psychiatry, 151, 494-498.

Wilhelm, F.H., y Roth, W.T. (1997). Clinical characteristics of flight phobia. Journal of Anxiety Disorders, 11, 241261. 